\title{
STD in children: syphilis and gonorrhoea
}

\author{
Sarah A Rawstron, Kenneth Bromberg, Margaret R Hammerschlag
}

\section{Introduction}

Sexually transmitted diseases, besides having an immediate impact on the infected individual, affects all sexual partners, and even the next generation. Infection in young children raises the possibility of sexual abuse, thus having legal as well as medical implications. Syphilis and gonorrhoea are in many ways the archetypal examples of sexually transmitted diseases. They were also thought to be under control, but AIDS and the spread of microbial resistance has reinforced their impact on the public health, and especially the health of children.

\section{Syphilis}

Children can be infected with Treponema pallidum either in utero (congenital syphilis) or in a manner similar to adults (acquired syphilis). Although congenital syphilis is far more common than acquired syphilis, it will be briefly mentioned, the emphasis being on acquired syphilis. Congenital syphilis is transmitted transplacentally from an infected mother to her foetus; it is not spread by sexual transmission. In contrast, acquired syphilis in children is almost always sexually transmitted, from abuse by an infected adult.

\section{Historical overview}

The transmission of syphilis to infants has been recognised since the first descriptions of the disease. However, there is a paucity of information about acquired syphilis in children.

\section{Congenital syphilis}

Gaspar Torella in 1497 wrote one of the first descriptions of syphilis in children, ${ }^{1}$ but at that time transmission was felt to be from the wet nurse. Although in the nineteenth century much work was done by Hutchinson, Diday and Fournier, who described the disease, the mode of transmission to infants was still not entirely clear. However, in 1905 Schaudinn and Hoffmann demonstrated the existence of $T$ pallidum microscopically, and in 1906, Wasserman developed a means of testing for infection with syphilis, using syphilitic liver as the antigen. These key observations finally demonstrated that congenital infection only occurred when the mother was infected, albeit often asymptomatically. Although arsenicals and bismuth had been used successfully in some cases to prevent congenital syphilis, the introduction of penicillin in 1943 was the last important development which finally led to a decline in syphilis and congenital syphilis.

\section{Acquired syphilis}

Information from studies on acquired syphilis in children published in 1938 and 1939 contain most of the historical data available. Waugh in $1938^{2}$ described 35 children with acquired syphilis seen in a five year period in one clinic. The number of acquired cases was 35 (16\%) compared with $183(84 \%)$ congenital cases seen in the same hospital at the same time. There were 24 children who were between five months and 11 years and 11 children who were between 12 and 14 years of age. One child had primary syphilis, eight had secondary syphilis and 15 had early latent syphilis. The children older than 12 had all acquired syphilis from sexual contact, whereas the authors considered that only two of the younger children definitely acquired syphilis through sexual contact, with another child possibly having acquired syphilis sexually. The authors considered that the rest of the children had acquired syphilis from contact with a parent or sibling. The younger children's mothers all had early syphilis, and eight of the 10 fathers tested had syphilis. It is very probable that additional children had acquired syphilis through abuse, but many of the children were too young for a history to be obtained. Waugh ${ }^{2}$ agreed with Fournier who considered the mode of acquisition of syphilis in children to be "parturition, breast feeding (infected mother or wet nurse), hand feeding, drinking utensils, cups, spoons, nipples, pacifiers, toilet articles, family relations, kissing, objects sucked, whistles, trumpets, toys, especially when purchased from street vendors".

$S_{\text {mith }}^{3}$ reviewed the case histories of 125 children with acquired syphilis who had been seen in 19 hospitals. There were 52 children who were less than five years and 73 children who were between five and 10 years of age. Acquired syphilis was more common in girls than boys by about 2 to 1 . Primary syphilis occurred in 77 children, extragenital sites being involved in 31 . Thirty five children had secondary disease and four had latent infection. The descriptions of the chancres and signs of secondary syphilis were similar to those in adults, except that the chancres tended to be smaller. Smith determined transmission to be sexual in $43(34 \%)$, and non-sexual in $82(66 \%)$. The non-sexual transmission was: kissing (15); close contact \\ Pediatrics, SUNY \\ at Brooklyn, \\ S A Rawstron \\ M R Hammerschlag \\ Professor M R \\ Hammerschlag, Children's \\ Medical Center of Brooklyn, \\ Kings County Hospital
}


(14); transfusion (9), and no source recorded (44). It is very possible that those with no source were due to sexual abuse, since some of these children had genital chancres which were almost certainly caused by sexual abuse.

Historically, children have been described with genital chancres and extragenital chancres. ${ }^{3}$ The extragenital sites described were lip, mouth, tongue, tonsil, face and nose, neck, sternum and thigh.

\section{Epidemiology}

Almost all preadolescent children with syphilis have acquired syphilis from adults, therefore the epidemiology of adult syphilis is important in understanding syphilis in children. The epidemiology of both congenital and acquired syphilis in children is really the epidemiology of syphilis in adults. In the USA, syphilis in adults is usually associated with lower socio-economic status, race (black $>$ hispanic $>$ white) and living in an urban area. However, since 1986, there has been a tremendous rise in all forms of syphilis seen in the United States, associated with important epidemiologic changes. ${ }^{4}$ The incidence of primary and secondary syphilis increased from 13.7 to 18.4 cases per 100000 persons, with the greatest increases among black women and black men. In many areas, the rates were highest in cities. This increase in syphilis has been associated with the use of crack/cocaine, and consequent "sex for drugs" which has made contact tracing and treatment difficult since much of the sexual activity is anonymous. Numbers of babies with congenital syphilis have risen dramatically. In New York City cases of congenital syphilis rose by $500 \%$ from 57 cases in 1986 to 357 cases in 1988.5

Adolescents with syphilis have a similar epidemiology to adults. In addition, one third of the adolescents in one study 6 had a concomitant sexually transmitted disease in the recent past or when seen. A significant number $(48 \%)$ were "troubled, delinquent, or homosexual". Therefore, syphilis may be an indication of significant social problems in these adolescents.

In the United Kingdom, there are very few cases of congenital syphilis. There were four cases of congenital syphilis in children under two years of age in 1984, three in 1985 and nine in 1986. Since 1983 there have been about 100 cases per year of congenital syphilis in children aged 2 and over. ${ }^{7}$

Congenital syphilis remains a significant problem in the developing world, in particular most of Africa. The prevalence of positive nontreponemal tests in pregnant women ranges from $4 \%$ (Rwanda) to $15 \%$ (Zambia), in major urban areas. ${ }^{8}$ Unpublished reports also indicated that syphilis seropositivity among pregnant women in other areas of Africa was often $10 \%$ or more. $^{8}$ One study in Zambia found that nearly $1 \%$ of babies who were delivered at one hospital had signs of congenital syphilis at birth, ${ }^{9}$ and that $40 \%$ of stillbirths could be attributed to congenital syphilis. Thus congenital syphilis is a major cause of morbidity and mortality in much of Africa.

\section{Routes of transmission}

Transplacental Congenital syphilis results from transplacental infection of the developing foetus from an infected mother with spirochaetaemia. An untreated syphilitic pregnant woman may transmit infection to the foetus at any clinical stage of her disease, although transmission is more likely with early infection. Fiumara ${ }^{10}$ found that $50 \%$ of mothers with untreated primary or secondary syphilis had babies with congenital syphilis with another $50 \%$ being premature, neonatal deaths or stillbirths. The transmission to the foetus declined to $40 \%$ in early latent infection, and $10 \%$ in late latent syphilis. ${ }^{10}$

Perinatal Perinatal transmission can undoubtedly occur, from contact of the baby with primary or secondary genital lesions during delivery. However, this is extremely uncommon, almost all cases of syphilis in the newborn period being due to transplacental infection.

Accidental Transmission of syphilis requires rather intimate skin to skin contact, and treponemes have not been demonstrated to penetrate unbroken, keratinized epidermis. Accidental transmission is possible via breastfeeding, kissing and handling, although infection is very rarely transmitted by "casual" contact, and seems to require intimate skin to skin contact.

Sexual abuse Transmission of syphilis beyond the neonatal period is almost always venereal, via sexual abuse. Nowadays, almost all syphilis cases in children are felt to be acquired by sexual contact. The discrepancy between the historical and modern data probably reflects the heightened awareness of sexual abuse, and more extensive investigation of cases of syphilis in children. Syphilitic chancres in extragenital sites may possibly be from nonsexual contact, ${ }^{11}$ but it is unwise to dismiss sexual abuse as a cause of transmission of syphilis, even when there is no specific history of abuse given by the child. ${ }^{12}$

The prevalence of syphilis among children suspected of having been sexually abused is lower than some other sexually transmitted diseases such as gonorrhoea. White ${ }^{13}$ detected six cases among 108 of 409 prepubertal children on whom serologic tests were performed, in comparison with 46 children who had positive gonorrhoea cultures. Five children were symptomatic and had additional sexually transmitted diseases, and only one was symptomatic with chancres. DeJong ${ }^{14}$ found only one out of 532 abused children had a positive serologic test for syphilis. These numbers indicate that syphilis is not very common among children who have been abused, and is seen less often than it was in the pre-antibiotic era. ${ }^{2}$

Voluntary sexual intercourse Consenting sexual 
activity among adolescents is responsible for some cases of acquired syphilis in children. Most adolescents with syphilis have acquired their disease through consenting sexual activity, but sexual abuse should still be considered as a possibility, since Silber ${ }^{6}$ found that $10 \%$ of 33 adolescents (ages 12-19) were found to be victims of sexual abuse, and Vermund ${ }^{15}$ similarly found that $8 \%$ of males and $32 \%$ of females with syphilis or gonorrhoea at a detention facility had a history of sexual abuse.

\section{Clinical Presentation}

Congenital syphilis Congenital syphilis is arbitrarily defined as early (presentation less than 2 years of age), and late (presentation after 2 years of age). The presentation of early congenital syphilis is variable. Some babies are so severely affected that they are stillborn, and some die in the early neonatal period, despite antibiotics. However, not all babies will become symptomatic. The classic presentation of congenital syphilis is a baby with rhinitis (snuffles), a maculopapular rash and hepatosplenomegaly. ${ }^{1617}$ Typical $\mathrm{x}$-ray findings associated with congenital syphilis are present in $50-95 \%$ of babies with congenital syphilis ${ }^{16}{ }^{17}$ and consist of osteochondritis, periostitis and metaphyseal changes in the long bones, with femur and humerus being most often involved. Babies may also have neurosyphilis as manifest by a positive CSF VDRL on lumbar puncture. Other associated findings include haemolytic anaemia, hepatitis and nephrotic syndrome. ${ }^{16}$ Less severely affected babies may not have any obvious abnormal physical findings, but merely have positive $x$-ray findings, CSF VDRL or specific IgM tests as evidence of infection.

The outcome of early congenital syphilis varies according to the severity of disease at presentation. Stillbirth is an important outcome of congenital syphilis, and is seen in an estimated $25-50 \%$ of infections. 1018 Perinatal death used to occur in $25-30 \%$ of infections, 1019 but is much less common now, since many deaths in the past were due to prematurity. The outcome in babies who survive appears to be good. However, the long term outcome of neurosyphilis in these children is not well known, and in the past there were some children who developed mental retardation as a consequence of neurosyphilis.

Late congenital syphilis is very uncommon nowadays. The characteristics of late congenital syphilis include: Hutchinson's Triad (Hutchinson's teeth, interstitial keratitis and eighth nerve deafness); bone changes, such as frontal bossing, saddle nose, sabre tibia and perforation of the hard palate; Clutton's joint; and rhagades. ${ }^{20}$

Acquired syphilis The presentation of acquired syphilis in children is the same as that in adults. They present with primary chancres, secondary syphilis, or latent syphilis with only positive syphilis serology as evidence of infection. Of course, children with congenital syphilis may be seen for the first time after the neonatal period with no signs or symptoms of congenital syphilis, but merely with a positive syphilis serology test. The distinction between congenital and acquired syphilis can be difficult in situations where the only evidence of $T$ pallidum infection is a positive syphilis serology, and there are no other signs or symptoms of infection.

Ginsburg ${ }^{21}$ described three children with acquired syphilis, one of whom presented with a primary chancre and two with rashes of secondary syphilis. Similar findings were described by Ackerman ${ }^{22}$ who reported on three abused children (ages 3-6) who presented with rashes or condylomata lata of secondary syphilis. There have been other case reports of acquired syphilis in children who presented with a lip chancre, ${ }^{11}$ secondary syphilis, ${ }^{23}$ and condylomata lata. ${ }^{12}$

Adolescents with syphilis in one study 6 presented most commonly with secondary syphilis $(58 \%)$ and less commonly with primary syphilis (18\%) and latent syphilis $(12 \%)$.

\section{Diagnostic methods}

Congenital syphilis The diagnosis of congenital syphilis relies on a combination of history, physical findings, radiology and laboratory tests. ${ }^{24}$ Definitive diagnosis of congenital syphilis is made when treponemes are detected either by dark-field or immunofluorescent techniques from a baby, or placenta. A presumptive (or compatible) diagnosis of congenital syphilis is made when a baby has a positive non-treponemal test, confirmed by a treponemal test, and either: typical physical findings; or positive x-rays; or a positive CSF VDRL; or hepatitis/haemolytic anaemia; or a positive $T$ pallidum IgM.

The presence of non-treponemal antibodies in a baby's serum does not necessarily imply congenital syphilis. Both nontreponemal and treponemal antibodies in the baby's serum may be passively transferred maternal antibodies (IgG class antibodies), and the presence of these in a baby simply implies maternal infection. Indeed, when screening serum at delivery to detect infection, maternal blood is the best specimen to send, since cord blood or infant's serum will detect only two thirds of babies at risk for congenital infection, whereas maternal blood will detect almost all babies at risk. ${ }^{25} \mathrm{~A}$ more specific test for congenital syphilis is a $T$ pallidum IgM test (19s FTA-ABS IgM, or western blot $\operatorname{IgM}$ ), since IgM detected in a baby is not of maternal origin.

Acquired syphilis Acquired syphilis in children is diagnosed in the same way as syphilis in adults. Syphilis is diagnosed by either detecting $T$ pallidum in lesions, or by detecting antibody against $T$ pallidum in serum. Syphilis serologic tests are either nontreponemal (for example VDRL, RPR) or treponemal (FTA, MHA-TP).

Primary syphilis is diagnosed preferably by performing a dark-field examination or direct fluorescent antibody test for $T$ pallidum 
(DFA-TP), on suspected lesions. ${ }^{26}$ Dark-field examinations are very useful in the setting of a sexually transmitted disease clinic, where the specimens can be examined quickly while the patient is still present. However, in the context of syphilis in children it is probably easier to perform a DFA-TP, since dark-field microscopes are not usually available in paediatric clinics, or offices. The slides from suspected lesions can be fixed in acetone, and the DFA-TP can either be performed in a local laboratory, or sent to a reference laboratory for review. In addition, serologic tests for syphilis should be sent, stating that a confirmatory treponemal test FTA or MHA-TP should be performed if the nontreponemal serologic test for syphilis is negative.

Secondary syphilis is usually diagnosed by a positive serological test for syphilis, combined with signs and symptoms of secondary syphilis. If there are moist skin or mucous membrane lesions present, then a dark-field examination may also be performed, since these lesions contain many spirochaetes. A syphilis serology should always be performed in individuals with pityriasis rosea since secondary syphilis can be confused with this skin disorder.

Latent syphilis is diagnosed when there are no signs or symptoms of syphilis, but there is a positive non-treponemal test for syphilis which is confirmed by a treponemal test. Latent syphilis is divided into early latent (syphilis of less than one year's duration), and late latent (syphilis of more than one year's duration) in the United States of America. In the United Kingdom, latent syphilis is defined as early if less than two years duration, and late if more than two years duration. It is only possible to make the diagnosis of early latent syphilis if there is documentation of a negative syphilis serologic test within the year (or two) before diagnosis. Otherwise, the patient should be considered to have late latent syphilis.

The Centers for Disease Control (CDC) ${ }^{24}$ recommend that patients with late latent syphilis should ideally have a CSF examination. Lumbar puncture is particularly indicated in patients with: neurological signs and symptoms; serum nontreponemal titre $\geqslant$ 1:32; non-penicillin therapy planned; positive HIV antibody test.

It may also be difficult to decide if a child has congenital or acquired syphilis, if the only indicator of infection is a positive serological test. Clinicians dealing with these problems have to use information such as maternal history of syphilis, height of nontreponemal titre and history of abuse in order to try to determine whether the syphilis is congenital or acquired. The $\mathrm{CDC}^{24}$ recommend that children who present with syphilis after the newborn period have a lumbar puncture performed to rule out congenital syphilis.

However, CSF abnormalities are also common in early syphilis. Lukehart ${ }^{27}$ found that adults with primary and secondary syphilis had $T$ pallidum isolated from the CSF in $12 / 40$ (30\%). Most patients who had
$T$ pallidum isolated had other CSF abnormalities (increased CSF wbc, protein, or positive CSF VDRL), although no single CSF abnormality was associated with $T$ pallidum isolation. There were four patients who had no abnormal CSF findings but who had $T$ pallidum isolated from the CSF. These findings indicate that abnormalities in the spinal fluid are very common in early syphilis. However, it is not recommended that lumbar punctures be routinely performed in primary or secondary syphilis, since CNS involvement could be considered to be normal in early syphilis infection.

\section{Treatment and follow-up regimes}

Congenital syphilis Congenital syphilis presenting in the neonatal period should be treated with either aqueous crystalline penicillin G 50,000 units $/ \mathrm{kg} /$ dose intravenously every 8 to 12 hours, or 50,000 units $/ \mathrm{kg} /$ dose procaine penicillin once daily intramuscularly, both regimens given for 10-14 days. ${ }^{24}$ If the baby is at risk for congenital syphilis, has a negative evaluation, but the follow up cannot be assured, some physicians recommend giving a single dose of benzathine penicillin, 50,000 units $/ \mathrm{kg}$ intramuscularly. However, other experts recommend giving a full 10 day course of antibiotics to these children, because of some reported failures with benzathine penicillin in neonates. ${ }^{28}$

After the newborn period, children with syphilis should have a lumbar puncture performed to exclude neurosyphilis. Children with congenital syphilis should be treated with aqueous penicillin G 50,000 units $/ \mathrm{kg}$ /dose every 4-6 hours intravenously for 10-14 days. ${ }^{24}$ An alternative regimen that was previously recommended ${ }^{29}$ was procaine penicillin 50,000 units $/ \mathrm{kg} /$ dose, given intramuscularly once daily for 10 days.

Follow up of these children should consist of repeat nontreponemal antibody titres at $1,2,3,6$ and 12 months. If the antibody titres remain stable or rise significantly, then retreatment should be considered. Children with positive CSF VDRL should have repeat lumbar punctures at six monthly intervals until the CSF VDRL is non-reactive. A reactive CSF VDRL at six months is an indication for re-treatment.

Acquired syphilis Treatment of acquired syphilis in children is determined by the presenting stage of syphilis.

Primary, secondary or early syphilis ( $<1$ year duration) Children with this presentation should be treated with benzathine penicillin G, 50,000 units per kilogram intramuscularly, up to the adult dose of 2.4 million units. ${ }^{24} 30$ Children less than 12 years old with a history of penicillin allergy should undergo skin testing with penicillin desensitization and treatment if necessary. Children older than 12 years who have a history of penicillin allergy may be treated with tetracycline or erythromycin as alternatives. The adult dose of tetracycline is $500 \mathrm{mg}$ four times a day, or 
doxycycline $100 \mathrm{mg}$ twice daily, for two weeks. The dose of erythromycin that is recommended for adults is $500 \mathrm{mg}$ four times a day for 2 weeks.

Late latent syphilis ( $>1$ year duration) Children who present with late latent syphilis of more than one year's duration should be treated with three doses of benzathine penicillin, 50,000 units per kilogram per dose (maximum 2.4 million units per dose), at weekly intervals for three consecutive weeks. In the UK, procaine penicillin has been the preferred therapy, the dose is 600,000 units daily intramuscularly for 15 days. Children less than 12 years old with a history of penicillin allergy should be skin tested, desensitised and treated with pencillin if necessary. Children older than 12 years with a history of penicillin allergy may be treated with tetracycline (adult dose $500 \mathrm{mg}$ four times a day orally) or doxycycline (adult dose $100 \mathrm{mg}$ twice a day) for 4 weeks. The tetracycline regimens can only be given if neurosyphilis has been excluded by lumbar puncture.

Children with neurosyphilis found by lumbar puncture should receive aqueous pencillin G 200,000 to 300,000 units $/ \mathrm{kg} /$ day $(50,000$ units/kg dose every 4 to 6 hours) for 10 to 14 days in doses not to exceed the adult dose of 2 to 4 million units $(1.2 \mathrm{~g}-2.4 \mathrm{~g})$ every 4 hours. ${ }^{30}$ Some authorities ${ }^{30}$ also recommend following this with benzathine penicillin $G$ 50,000 units $/ \mathrm{kg}$ (maximum $2 \cdot 4$ million units) at weekly intervals for 3 consecutive weeks.

Follow up of these children should consist of repeated nontreponemal serology tests performed at 1,2,3, 6 and 12 months. If the nontreponemal antibody titres have not decreased fourfold by three months (primary and secondary syphilis); six months (early latent syphilis); or one year (late latent syphilis), then the patient should be reevaluated and retreated. ${ }^{24}$

It is recommended that all patients with syphilis be counselled about the risks of HIV and be encouraged to have HIV testing performed. ${ }^{24}$

Future Developments The interaction of syphilis and HIV disease has received much recent attention. ${ }^{31} \mathrm{HIV}$ disease in adults has been associated with treatment failures in patients with syphilis, and more rapid progress of syphilis in others. There have been no data published on the interaction between HIV and syphilis in children, either congenital or acquired. One may assume that any problems seen in adults with HIV and syphilis may be seen in acquired syphilis in children. Further study may lead to changes in recommended doses of antibiotics used to treat syphilis in HIV patients.

There has been research on many aspects of the diagnosis of syphilis, particularly congenital syphilis. Tests undergoing development are IgM tests (Western Blot ${ }^{32}$ ), and also polymerase chain reaction (PCR). ${ }^{33}$ PCR may be useful in the future in the diagnosis of neurosyphilis.

\section{Gonorrhoea \\ History}

Gonorrhoea is one of the oldest known human illnesses. Although the causative organism was first described by Neisser in 1879 , there are references to venereal urethritis in ancient Chinese writings, the Old Testament and other ancient texts. Galen (AD 130) introduced the term gonorrhoea ("flow of seed") due to confusion of the urethral discharge with semen. The organism was first cultivated in 1882 by Leistikow and Loeffler. Gonococcal ophthalmia neonatorum was described at that time. Shortly afterwards came the studies of Credé. Credé realised that gonococcal ophthalmia neonatorum was acquired by the infant perinatally during passage through the infected birth canal. He demonstrated that neonatal ocular prophylaxis with $2 \%$ silver nitrate reduced the incidence of gonococcal ophthalmia from $10 \%$ to $0.3 \%$ of live births. ${ }^{34}$ Genital gonococcal infection in children, specifically vulvovaginitis, was also recognised during this period. Although transmission in several hospital associated outbreaks was thought to have occurred through fomites, including contaminated bed clothes and undergarments, the role of sexual assault was also becoming recognised.

\section{Epidemiology}

Infections in pregnancy and the neonate

Neisseria gonorrhoeae is one of the most prevalent sexually transmitted organisms. The rates of maternal gonorrhoea in most U.S. populations is $<5 \%$ whereas the rates in some parts of Africa may exceed $10 \%$. Maternal gonococcal infection has been associated with a statistically greater occurrence of premature rupture of membranes, prolonged rupture of membranes, choriomnionitis and premature delivery. ${ }^{35}$ Infants can acquire the infection from their mothers during delivery and develop gonococcal ophthalmia, which can cause serious eye damage. Rarely, infants may develop disseminated gonococcal infection with arthritis. Ophthalmia neonatorum due to $N$ gonorrhoeae used to be a leading cause of blindness. The introduction of the practice of prenatal screening and treatment of pregnant women as well as neonatal ocular prophylaxis has dramatically affected the rates of gonococcal ophthalmia in most industrialised countries. ${ }^{36}$ The most frequent infectious cause of neonatal ophthalmia in the United States is now Chlamydia trachomatis. The incidence of gonococcal ophthalmia is estimated to be $0.04 \%$, whereas the incidence of chlamydial ophthalmia is estimated to be $0.4 \% .{ }^{36}{ }^{37}$ However, studies from Kenya have found that $N$ gonorrhoeae was responsible for $43 \%$ of cases of ophthalmia neonatorum, $C$ trachomatis for $13 \%$ and both organisms for 4\%. ${ }^{38}$ The risk for an infant born to a mother with untreated gonorrhoea of developing gonococcal ophthalmia appears to be approximately $30 \% .{ }^{35}$ Laga et al from Kenya found the incidence of gonococcal ophthalmia in 
infants born to untreated mothers to be $42 \% .{ }^{39}$

\section{Infection in children}

$N$ gonorrhoeae is the commonest reported sexually transmitted disease found in sexually abused children in the U.S., with prevalences ranging from 3 to over $25 \% .131440$ Since many children with gonococcal infection present to the physician with a complaint of vaginal or urethral discharge rather than one of sexual assault, these numbers may not reflect the actual risk of infection. The risk to the child of acquiring gonorrhoea after sexual assault depends on a number of factors including the prevalence of gonococcal infection among adults in the population, and the type and duration of the assault. 4041 Two recent studies of the risk of acquiring a sexually transmitted disease after sexual assault in postpubertal females found the risk of acquiring gonorrhoea to be 0 to $4 \cdot 2 \% .4243$ In contrast, the risk of acquiring trichomoniasis and bacterial vaginosis ranged from $12 \%$ to over $20 \%$. Both studies included a large number of adolescent women aged 16 to 20 years. Gonorrhoea in prepubertal children occurs most often in females; vulvovaginitis accounts for about $75 \%$ of gonococcal disease in this age group. However as many as $50 \%$ of vaginal infections may be asymptomatic. ${ }^{14}$ Children are frequently infected at multiple sites. Rawstron et al $\mathbf{4 4}$ reported eight girls with gonococcal infection, all were infected in the vagina and rectum and two $(25 \%)$ had pharyngeal infection. The pharyngeal infections and all but one of the rectal infections in these children were asymptomatic. Similar findings have been reported in other series.1314 Routine culturing of the oropharynx for $N$ gonorrhoeae in abused children and their siblings may uncover some unsuspected sexual abuse cases. ${ }^{45} 46$ There are limited data on the prevalence of gonococcal infection among children in developing countries, although it appears to be a fairly frequent cause of vulvovaginitis among girls in Africa. ${ }^{47}$

\section{Infection in adolescents}

Reports from the United States have found gonorrhoea in $3 \%$ to $12 \%$ of sexually active adolescents of both sexes. Data from Great Britain are very similar. ${ }^{48}$ Coinfection with Chlamydia trachomatis is frequent and may be as high as $50 \%$ in some populations. The prevalence of chlamydial infection has generally been higher than that for gonorrhoea in most of the adolescent populations studied in the United States

\section{Routes of transmission}

Perinatal Transmission of $N$ gonorrhoeae from mother to infant occurs during delivery. Premature rupture of membranes prior to delivery appears to increase the risk of the infant acquiring gonococcal infection. ${ }^{35}$ The infant may aspirate infected vaginal secretions leading to nasopharyngeal infection and potential systemic dissemination leading to sepsis or arthritis. The organism can also be directly inoculated into the conjunctivae. Gonococcal infection of scalp wounds that occur during foetal monitoring resulting in scalp abscess has also been described.

\section{Sexual abuse}

A positive culture for $N$ gonorrhoeae from any site in a child without prior peer sexual activity is strongly suggestive of sexual abuse. ${ }^{40}$ Gonococcal infection can be spread by sexual play among children, but the infection probably was acquired originally through sexual abuse. ${ }^{49}$ The question of whether gonococcal infection in children can be acquired through fomites still arises although there are no convincing data for any nonsexual mode of transmission. ${ }^{50}$ The most convincing epidemic of gonococcal infection among children in which spread by fomites probably occurred was reported in 1927.51 It involved 67 of 182 infants in a single hospital ward during a one month period. Contaminated rectal thermometers were thought to be the source. In a commentary written in 1979, Gellis raises an important point: "You have to wonder what transpired in that hospital ward".52 Although there have been isolated reports of children who developed gonococcal ophthalmia and vaginitis, where cosleeping with an infected adult was the only perceived risk, the possibility of sexual abuse was not even discussed. There have also been several outbreaks among institutionalised children reported in the preantibiotic era, but sexual play, again, could not be excluded as a possible source. Certainly we do not try to explain away gonococcal infections in sexually active adults or adolescents as being acquired through contact with inanimate objects. Spread by contaminated towels may not even be anatomically feasible since the vagina is an internal structure. Spread by fomites does not explain the frequent occurrence of pharyngeal gonorrhoea in children who have been victims of sexual abuse.

Branch and Paxton ${ }^{53}$ demonstrated that careful interviewing can lead to a history of sexual contact in almost all children with gonococcal infection who are over one year of age. Usually the type of sexual activity correlated with the site of infection, that is, the presence of pharyngeal infection was associated with a history of orogenital contact. Farrell et al ${ }^{54}$ were able with extensive interviewing and routine culturing of contacts to identify the source of the gonococcal infection in $83 \%$ of the children they evaluated. However failure to identify an infected perpetrator does not rule out the possibility of sexual abuse, since treatment may render an individual culture negative within hours of therapy. Since treatment may be obtained confidentially, the suspect may be treated before being cultured.

\section{Voluntary sexual intercourse}

Consenting sexual activity remains responsible for the vast majority of cases of gonorrhoea among adolescents in the United 
States. However, a number of adolescents are also victims of sexual abuse. ${ }^{6} 15$

\section{Clinical presentation \\ lnfection in infants}

Neonatal gonococcal ophthalmia usually starts as an initially nonspecific conjunctivitis, two to three days after birth. A thick purulent exudate may rapidly develop and corneal ulceration, perforation and iridocyclitis may develop if therapy is not started promptly. The incubation period can be longer and may overlap with $C$ trachomatis. Fransen et al 38 found that in $9 \%$ of the cases of gonococcal conjunctivitis they studied, the disease started after 14 days of age. Several of these infants had received some antibiotic therapy and it was thought that the late presentation represented a relapse due to inadequate treatment.

\section{Infection in children}

$N$ gonorrhoeae may cause purulent vulvovaginitis in girls or urethritis in boys. Gonococcal ophthalmia may also occur due to autoinoculation from a genital site. However, as many as $20-25 \%$ of children with genital cultures containing $N$ gonorrhoeae may be asymptomatic and an even higher number of rectal and pharyngeal infections are asymptomatic. 144044 The vaginal discharge when present may be minimal and confused with a benign discharge. Symptoms referrable to the urinary tract may predominate. Ascending pelvic infection may also occur in prepubertal females and may occur in the absence of significant vaginal discharge. Anorectal gonorrhoea in general produces only a minor inflammatory reaction with local burning and discharge, but many patients will be asymptomatic.

\section{Infection in adolescents}

Gonococcal infections in sexually active adolescents can present as cervicitis, urethritis, proctitis, epididimitis, disseminated gonococcal infection (DGI), perihepatitis (Fitz-HughCurtis syndrome) and salpingitis. Salpingitis is the most serious potential complication of gonorrhoea in adolescent women. Approximately $15 \%$ of teenagers who develop gonorrhoea will progress to pelvic inflammatory disease (PID). PID in adolescents is particularly likely to result in infertility, and PID is the single most common cause of infertility in young women. Diagnosing PID may be difficult and the differential diagnosis includes numerous other conditions of the lower abdomen, including appendicitis, ectopic pregnancy, cholecystitis, mesenteric adenitis, pyelonephritis, and septic abortion. Misdiagnosis of PID is common and is one of the more common causes of medically nonindicated laparotomy. Laparoscopy may assist in establishing a diagnosis. Shafer et al 55 suggest that a clinical diagnosis of PID in adolescents be supported by the presence of lower abdominal pain and tenderness, cervical motion tenderness, and adnexal tenderness. Fever, leukocytosis, elevated sedimentation rate, and adnexal mass on abdominal ultrasound support the diagnosis. Culdocentesis, if performed, may reveal evidence of purulent reaction in the peritoneal cavity.

\section{Diagnostic methods}

The simplest method of diagnosis is a Gram stain of urethral discharge, the gonococci appear as Gram negative, biscuit-shaped intracellular diplococci. Gram stains of synovial fluid, urethral discharge, or urine sediment in the male may reveal the characteristic organism. In the adult female the Gram stain is less reliable since the cervix may be colonized with other Neisseria species.

In younger children, because of the medico-legal implications, the importance of an accurate microbiologic diagnosis cannot be overemphasised. Culture is the preferred method. Gram stain of the vaginal discharge in a child with suspected gonorrhoea may reveal Gram-negative intracellular diplococci, but may be misleading since normal flora will often contain other Neisseria species or Moraxella which are also Gram negative diplococci.

Cultures of pharynx, rectum and vagina/urethra should be taken and immediately plated onto media appropriate for isolation of $N$ gonorrhoeae. The media that may be used include chocolate blood agar and Thayer-Martin media. The plates should then be placed in an atmosphere enriched with $\mathrm{CO}_{2}$, the easiest method being an extinction candle jar. The isolation of Gram-negative, oxidase positive diplococci should be confirmed with additional tests which include rapid carbohydrate tests, enzyme-substrate tests, rapid serological tests or DNA probes. Failure to perform appropriate confirmatory tests may lead to misidentification of other organisms as $N$ gonorrhoeae. Whittington et al 56 found that fourteen of 40 presumptive gonococcal isolates from children sent to the CDC in Atlanta, for confirmation, had been misidentified as $N$ gonorrhoeae. They included other Neisseria species, Moraxella catarrhalis and Kingella dentrificans. The CDC recommends that confirmation of an organism as $N$ gonorrhoeae from a child should include at least two procedures that use different principles, for example, biochemical and enzyme-substrate or serologic.

Currently there are also several non culture diagnostic methods available for detection of $N$ gonorrhoeae including enzyme immunoassays (EIA) and DNA probes. Tests based on PCR are also under development. These assays may be appropriate for screening adolescents, but cannot be used in children, again because of medico-legal issues. Preliminary data suggest that although the specificity of these tests may be high, they are not $100 \%$ specific when compared with culture. Another potential problem is that these assays do not provide any information on antimicrobial susceptibility.

\section{Therapy}

The treatment of gonococcal infections in the 
United States is influenced by the spread of infections due to antibiotic resistant $N$ gonorrhoeae, including penicillinase producing $N$ gonorrhoeae (PPNG), tetracycline-resistant $N$ gonorrhoeae (TRNG) and strains with chromosomally mediated resistance to multiple antibiotics. Data from the U.S. and Singapore have shown that the prevalence of infections due to PPNG in children parallels that in adults, which is not surprising since most gonococcal infections in infants and children are acquired from adults. 4457 Therapy is also affected by the high frequency of chlamydial co-infections in persons with gonorrhoea, which is especially a problem in adolescents.

The susceptibility of $N$ gonorrhoeae to various antibiotics has been shown to change over time in any locality. Surveillance is very important and requires regular testing of recent clinical isolates and all isolates associated with treatment failure. The CDC defines any locality with a prevalence of infections due to PPNG $>3 \%$ as a hyperendemic area and recommends that a penicillinase resistant antibiotic be used as a first line drug. ${ }^{24}$ Rawstron et al 44 reported from Brooklyn, N.Y. that $26.5 \%$ of isolates of $N$ gonorrhoeae from infants and children seen over a one year period were penicillin resistant. During the same period $25.3 \%$ of the isolates from adults were PPNG.

Infants born to mothers with untreated gonorrhoea are at high risk of infection (ophthalmia and DGI) and should receive a single intramuscular (IM) or intravenous (IV) dose of ceftriaxone $(50 \mathrm{mg} / \mathrm{kg}$ up to $125 \mathrm{mg})$ or cefotaxime $(100 \mathrm{mg} / \mathrm{kg})$. For the treatment of gonococcal ophthalmia, the CDC currently recommends ceftriaxone, 25 to $50 \mathrm{mg} / \mathrm{kg} /$ day up to $125 \mathrm{mg}$ IV or IM in a single daily dose for 7 days. However, data from Kenya ${ }^{58}$ suggest that a single dose of ceftriaxone is more than adequate. The World Health Organisation recommended in 1986 the use of either cefotaxime, $100 \mathrm{mg} / \mathrm{kg}$ as a single IM dose or kanamycin $25 \mathrm{mg} / \mathrm{kg}$ as a single IM dose plus $1 \%$ tetracycline or $0.5 \%$ erythromycin ophthalmic ointment for ten days. ${ }^{59}$ The use of ceftriaxone or cefotaxime does not require additional topical therapy which significantly simplifies therapy. Infants with gonococcal ophthalmia should also receive eye irrigation with buffered saline until the discharge is cleared, usually for 24 hours. Topical antibiotic therapy is not indicated. Infants should also be examined by an ophthalmologist to ensure corneal perforation has not occurred.

Some infants may also have co-infection with $C$ trachomatis. Clinically it can be difficult to differentiate chlamydial from gonococcal conjunctivitis in some infants. Chlamydial infection should be considered in those infants where the conjunctivitis persists despite appropriate therapy. Chlamydial cultures or an antigen detection test should be obtained and the infant be treated with erythromycin suspension, $50 \mathrm{mg} / \mathrm{kg} /$ day, orally for two weeks.
Children with genital, rectal or pharyngeal gonorrhoea may also be treated with ceftriaxone. Children who weigh $\geqslant 45 \mathrm{~kg}$ should receive a single IM dose of $250 \mathrm{mg}$, if $\leqslant 45 \mathrm{~kg}, 125 \mathrm{mg}$. This regimen is very effective in eliminating $N$ gonorrhoeae from the pharynx and rectum. ${ }^{44}$ Children aged over 2 years with known hypersensitivity to betalactam antibiotics may be treated with spectinomycin, $40 \mathrm{mg} / \mathrm{kg}$ IM, once. Spectinomycin is not as effective in rectal or pharyngeal gonorrhoea. Follow up cultures should be obtained from these sites. Cefotaxime should also be effective, as it is in adults, but there are no reported studies of its use in treating children with gonorrhoea.

Children with DGI (bacteraemia or arthritis) should be treated with ceftriaxone $50 \mathrm{mg} / \mathrm{kg}$ (maximum 1 gram) once daily for seven days. All children with rectogenital gonorrhoea should also be evaluated for coinfection with chlamydia. If the infection is known not to be PPNG, one can use amoxicillin, $50 \mathrm{mg} / \mathrm{kg}$ orally as a single dose plus probenicid, $25 \mathrm{mg} / \mathrm{kg}$. The newer expanded spectrum oral cephalosporins such as cefixime have been shown to be effective as a single oral dose in adults, but have not yet been evaluated in children.

Single-dose efficacy is a major consideration in the treatment of gonococcal infections in adolescents. Another important factor is coinfection with $C$ trachomatis, which can be documented in up to $45 \%$ of adolescents with gonorrhoea in some populations. For teenagers $\geqslant 12$ years, treatment should follow the recommended regimens for adults. The first-line regimen recommended by the CDC is single dose ceftriaxone, $250 \mathrm{mg}$ IM plus doxycycline, $100 \mathrm{mg}$ orally twice daily for seven days. The latter is necessary to treat possible co-infection with $C$ trachomatis.

Alternative regimens for individuals who cannot tolerate ceftriaxone are spectinomycin, $2 \mathrm{~g}$ IM in a single dose, plus doxycycline. Other alternatives are cefotaxime, $1 \mathrm{~g}$ IM once, cefuroxime axetil, $1 \mathrm{~g}$ orally plus $1 \mathrm{~g}$ probenicid, and ceftizoxime, $500 \mathrm{mg} \mathrm{IM}$ once. Experience is less extensive with these regimens. If the adolescent is $\geqslant 16$ years one could also use ciprofloxacin $500 \mathrm{mg}$ orally once or norfloxacin, $800 \mathrm{mg}$ orally once. All these regimens should be followed by a seven day course of doxycycline. Tetracycline cannot be used as a single drug for gonorrhoea and chlamydia because of the increasing prevalence of TRNG strains.

\section{Prevention of Ophthalmia Neonatorum}

Neonatal ocular prophylaxis to prevent gonococcal ophthalmia neonatorum is required by law in most of the United States. However, the use of neonatal ocular prophylaxis for the prevention of gonococcal ophthalmia is not universal, several Western countries including Great Britain and Denmark do not practise neonatal ocular prophylaxis. They depend instead on universal prenatal care and intensive contact tracing to control maternal and neonatal gonococcal infections. Recent expe- 
rience in the United States tends to support this as the highest risk of gonococcal ophthalmia appears to occur in infants born to mothers who have not received any prenatal care. ${ }^{60}$ Although prenatal screening and treatment of maternal gonorrhoea have a major effect on the prevention of neonatal gonococcal ophthalmia, neonatal ocular prophylaxis is still warranted in many populations because of poor prenatal care.

Laga et al ${ }^{39}$ reported from Kenya, where there was no programme of prenatal screening, that neonatal ocular prophylaxis with either tetracycline ophthalmic ointment or silver nitrate drops was very effective in preventing neonatal gonococcal ophthalmia in infants born to infected women. The incidence of gonococcal ophthalmia was reduced from $42 \%$ to $7 \%$ in those infants who received prophylaxis with silver nitrate and to $3 \%$ of those who received tetracycline ointment. The impact of prenatal care was underlined by the study of Hammerschlag et al which compared silver nitrate to tetracycline and erythromycin as ocular prophylaxis. ${ }^{60}$ Eight infants developed gonococcal ophthalmia during the study; seven of the mothers had no prenatal care and five were abusers of crack cocaine.

The regimens recommended by the CDC for ocular prophylaxis are erythromycin $(0.5 \%)$ ophthalmic ointment once; tetracycline $(1 \%)$ ophthalmic ointment once, or silver nitrate $(1 \%)$ aqueous solution, once. Bacitracin ointment is not recommended. One of the above preparations should be installed into the eyes of the neonate as soon as possible after delivery, and definitely within one hour of birth. Single-use tubes or ampoules are preferred and are available for erythromycin and silver nitrate. There is a single-dose tetracycline solution available, but there are no data on its efficacy for preventing gonococcal ophthalmia. Tetracycline and erythromycin also appear to be effective in the prevention of gonococcal ophthalmia due to TRNG and PPNG, probably due to the high concentration of the drug in these preparations. ${ }^{34}$ Neonatal ocular prophylaxis does not appear to be effective for the prevention of chlamydial conjunctivitis.

\section{Future Developments}

A major direction in research in gonococcal infection has been the development of a vaccine. Whether vaccination would be used in childhood is not clear. The development of rapid non culture methods will probably not be of use in children because of the medicolegal implications of gonococcal infection in young children. These tests may be very helpful in sexually active adolescents. Several companies are in the process of developing PCR techniques that will detect $N$ gonorrhoeae and $C$ trachomatis in a single specimen.

Treatment poses another set of problems. Infection due to PPNG and TRNG has become endemic in many areas, necessitating the use of more expensive, often parenteral antibiotics. Although the quinolone anti- biotics appear to be very effective as a single dose oral therapy for gonorrhoea in adults, these drugs cannot be used in children. There is also the real concern for the development of widespread quinolone resistance, which appears to have happened in the Far East. The new expanded spectrum oral cephalosporins appear to be effective as single dose therapy in adults but no studies have been conducted in children. Oral therapy is very desirable as it would be less traumatic to the child.

\section{Addendum}

Aqueous crystalline penicillin is available in Britain as benzyl penicillin $(50,000$ units $/ \mathrm{kg}=$ $30 \mathrm{mg} / \mathrm{kg}$ ).

Procaine penicillin is available in Britain as Bicillin.

Benzathine penicillin is available in Britain as the oral formulation only.

Ceftriaxone, $0.5 \%$ erythromycin ophthalmic ointment, silver nitrate $1 \%$ aqueous solution and Bacitracin ointment are not available in Britain.

1 Schulz KF, Murphy FK, Patamasucon P, Meheus AZ. Congenital Syphilis. In: Holmes KK, Mardh P-A, Sparling PF, et al eds. Sexually Transmitted Diseases. New York, McGraw Hill, 1990:821-42, 2nd ed.

2 Waugh JR. Acquired syphilis in infancy and childhood. Am 7 Syph Gon Ven Dis 1938;22:607-22.

3 Smith FR. Acquired syphilis in children. Am 7 Syph Gon Ven Dis 1939;23:165-85.

4 Rolfs RT, Goldberg M, and Sharrar RG. Risk factors for syphilis: cocaine use and prostitution. Am $\mathcal{f}$ Public Health 1990;80:853-7.

5 Centers for Disease Control. Congenital Syphilis-New York City, 1986-1988. MMWR 1989;38:825-9.

6 Silber TJ, Niland NF. The clinical spectrum of syphilis in adolescence. Sex Active Teenagers 1988;2:93-7.

7 Public Health Laboratory Service Communicable Disease Surveillance Centre. Sexually transmitted disease in Britain: 1985-6. Genitourin Med 1989;65:117-21.

8 Schulz KF, Cates W Jr, O'Mara PR. Pregnancy loss, infant death, and suffering: legacy of syphilis and gonorinfant death, and suffering: legacy of syphilis and

9 Hira SK, Bhat GJ, Ratnam AV, Chintu C, Mulenga RC. Congenital syphilis in Lusaka. II. Incidence at birth and potential risk among hospital deliveries East Afr Med f 1982;59:306-10

10 Fiumara NJ, Fleming WL, Downing JG, Good FL. The incidence of prenatal syphilis at The Boston City Hospital. N Eng $\mathcal{F}$ Med 1952;247:48-52.

11 Aloi F. Lip syphilitic chancre in a child. Pediatr Dermatol 1987;4:63

12 Horowitz S, Chadwick DL. Syphilis as a sole indicator of sexual abuse: two cases with no intervention. Child Abuse and Neglect 1990;14:129-32.

13 White ST, Loda FA, Ingram DL, Pearson A. Sexually transmitted diseases in sexually abused children. transmitted diseases in

14 DeJong A. Sexually transmitted diseases in sexually abused children. Sex Transm Dis 1986;12:123-6.

15 Vermund SH, Alexander-Rodriguez T, Macleod S, Kelley $\mathrm{KF}$. History of sexual abuse in incarcerated adolescents with gonorrhoea or syphilis. $f$ Adol Health Care 1990;11:449-52.

16 Hira SK, Ganapati JB, Patel JB, et al. Early congenital syphilis: clinico-radiologic features in 202 patients. Sex Transm Dis 1985;12:177-83.

17 Ricci JM, Fojaco RM. O'Sullivan MJ. Congenital syphilis: the University of Miami/Jackson Memorial Medical Center experience, 1986-1988. Obstet Gynecol 1989, 74:687-93.

18 Wendel GD. Early and Congenital Syphilis. Obstet Gynecol Clin N Am 1989;16:479-94.

19 Ingraham NR. The diagnosis of infantile congenital syphilis during the period of doubt. Am $\mathcal{F}$ Syph Neurol 1935;19:547-80.

20 Fiumara HJ, Lessell S. Manifestations of late congenital syphilis. An analysis of 271 patients. Arch Dermatol !970;102:78-83.

21 Ginsburg CM. Acquired syphilis in prepubertal children Pediatr Infect Dis 1983;2:232-4.

22 Ackerman AB, Goldfadam G, Cosmides IC Acquired syphilis in early -hildhood. Arch Derm 1972;106:92-3.

23 Echols SK, Shupp DI , Schroeter AL Acquired secondary syphilis in a child. $¥$ A $1:$ Acad Dermatol 1990;23:313-4. 4 Centers for Disease Control. 1989 Sexually transin-4. diseases treatment guidelines. MMWR $1989 ; 38(\mathrm{~S}-8)$. 
25 Rawstron SA, Bromberg K. Comparison of maternal and newborn serologic tests for syphilis. Am $\mathcal{F}$ Dis Child 1991;145:1383-8.

26 Yobs AR, Hunter EF. Fluorescent antibody technique in early syphilis. Arch Pathol 1964;77:220-5.

27 Lukehart SA, Hook EW, Baker-Zander S, Collier AC Critchlow CW, Handsfield $\mathrm{HH}$. Invasion of the centra nervous system by Treponema pallidum: Implications for diagnosis and treatment. Ann Intern Med 1988;109: 855-62.

28 Beck-Sague C, Alexander ER. Failure of benzathine penicillin $\mathrm{G}$ therapy in early congenital syphilis. Pediat Infect Dis $\mathcal{F}$ 1987;6:1061-4.

29 Centers for Disease Control. Guidelines for the prevention and control of congenital syphilis. MMWR 1988; 37(S1):1-13.

30 Committee on Infectious Diseases, American Academy of Pediatrics. Report of the Committee on Infectious Diseases 1991:453-63.

31 Musher DM, Hamill RJ, Baughn RE. Effect of human immunodeficiency virus(HIV) infection on the course of syphilis and on the resp

32 Sánchez PJ, McCracken GH Jr, Wendel GS, Olsen K, et al. Molecular analysis of the fetal IgM response to Treponema pallidum antigens: implications for improved serodiagnosis of congenital infection. $\mathcal{f}$ Infect Dis 1989;159:508-17.

33 Sánchez PJ, Wendel GD, Grimprel E, et al. Diagnosis of congenital neurosyphilis by the rabbit infectivity test (RIT), and polymerase chain reaction (PCR). Pediatr Res 1991;29:286A.

34 Credé KSF. Die Verhütung der Augenentzünderdung der Neugeborenen. Arch Gynakol 1881;17:50-3.

35 Handsfield HH, Hodson WA, Holmes KK. Neonatal gonococcal infection: I. Orogastric contamination with Neisseria gonorrhoeae $7 A M A$ 1973;225:697-701.

36 Rothenberg R. Ophthalmia neonatorum due to Neisseria gonorrhoeae. Prevention and treatment. Sex Transm Dis 1979;6 (suppl): 189-91.

37 Hammerschlag, MR. Neonatal ocular prophylaxis. Pediatr Infect Dis $\mathcal{F} 1988 ; 7: 81-2$.

38 Fransen L, Nsanze $\mathrm{H}$, Klauss V, et al. Ophthalmia neonatorum in Nairobi, Kenya. The roles of Neisseria gonorrhoeae and Chlamydia trachomatis. F Infect Dis 1986; 153:862-9.

39 Laga M, Plummer FA, Piot P, et al. Prophylaxis of gonococcal and chlamydial ophthalmia neonatorum. $N$ Engl f Med 1988;318:653-7.

40 Schwarcz SK, Whittington WL. Sexual assault and sexually transmitted diseases: detection and management. Rev Infect

41 Glaser JB, Hammerschlag MR, McCormack WM Epidemiology of sexually transmitted diseases in rape victims. Rev Infect Dis 1989;11:246-254.
42 Jenny C, Hooton TM, Bowers BA, et al. Sexually transmitted diseases in victims of rape. $N$ Engl $f$ Med 1990;322:713-6.

43 Glaser JB, Schachter J, Benes S, et al. Sexually transmitted diseases in postpubertal rape victims. $\mathcal{F}$ Infect Dis 1991; 164:726-30.

44 Rawstron SA, Hammerschlag MR, Gullans C, et al. Ceftriaxone treatment of penicillinase-producing Neisseria gonorrhoeae infections in children. Pediatr Infect Dis $\mathcal{F} 1989 ; 8: 445-8$

45 Groothuis JR, Bischoff MC, Jauregui LE. Pharyngeal gonorrhoea in young children. Pediatr Infect Dis 1983;2. 99-101.

46 McClure EM, Stack MR, Tanner T, et al. Pharyngeal culturing and reporting of pediatric gonorrhoea in Connecticut. Pediatrics 1986;78:509-10.

47 Alausa KO, Osoba AO. Epidemiology of gonococcal vulvovaginitis among children in the tropics, $\mathrm{Br} \mathcal{F}$ Venereal Dis 1980;56:239-42.

48 Estreich S, Forster GE. Sexually transmitted diseases in children: Introduction. Genitourin Med 1992;68:2-8.

49 Potterat J, Markewich GS, King RD, et al. Child-to-child transmission of gonorrhea. Report of asymptomatic genital infection in a boy. Pediatrics 1986;78:711-2.

50 Neinstein LS, Goldenring J, Carpenter S. Non-sexua transmission of sexually transmitted diseases: An infrequent occurrence. Pediatrics 1984;74:67-75.

51 Cooperman MB. Gonococcus arthritis in infancy. $\mathrm{Am} f$ Dis Child 1927;33:932-48.

52 Gellis S. Prepubertal gonorrhea. Pediatric Notes 1979; 3:1.

53 Branch G, Paxton R. A study of gonococcal infections among infants and children. Pub Health Rep 1965;80: 347-52.

54 Farrell MK, Billmire ME, Shamroy JA, Hammond JG. Prepubertal gonorrhea: A multidisciplinary approach Pediatrics 1981;67:151-3.

55 Shafer M-A, Irwin CE, Sweet RL. Acute salpingitis in the adolescent female. F Pediatr 1982;100:339-50.

56 Whittington WL, Rice RJ, Biddle JW, et al. Incorrect identification of Neisseria gonorrhoeae from infants and children. Pediatr Infect Dis $\mathcal{f}$ 1988;7:3-10.

57 Thirumoorthy T, Rajan VS, Goh CL. Penicillinase-producing Neisseria gonorrhoeae ophthalmia neonatorum in Singapore. Br $\mathcal{F}$ Venereal Dis 1982;58:308-10.

58 Laga M, Naamara W, Brunham RC, et al. Single-dose therapy of gonococcal ophthalmia neonatorum with ceftherapy of gonococcal ophthalmia neonato

59 Lepage P, Kestelyn P, Bogaerts J. Treatment of gonococcal conjunctivitis with a single intramuscular injection of cefotaxime. Antimicrob Chemother 1990;26:Suppl.

60 Hammerschlag MR, Cummings C, Roblin PM, et al. Efficacy of neonatal ocular prophylaxis for the prevention of chlamydial and gonococcal conjunctivitis. $N$ Engl f Med 1989;32:769-72. 